\title{
Fast ReRouting Flow-based Model with Implementation of Path Protection
}

\author{
Yeremenko Oleksandra, \\ Yevdokymenko Maryna, \\ Sleiman Batoul, \\ Omowumi Stephen Olayinka
}

\author{
Kharkiv National University of Radio Electronics, 14 Nauky Ave, \\ Kharkiv UA-61166, Ukraine \\ oleksandra.yeremenko.ua@ieee.org, \\ maryna.yevdokymenko@ieee.org, \\ sleimanbatoul@hotmail.com, \\ iamengineerstephen@gmail.com
}

\begin{abstract}
In this paper, the fast rerouting model with the implementation of the path protection scheme in the infocommunication network is investigated. Within the proposed Fast ReRoute model with the path protection schemes, the problem of calculating the set of primary and backup disjoint paths was solved. The advantage of the improved model is the possibility of implementing protection schemes 1:1, 1:2, .., 1:n without introducing an additional set of routing variables. This results in reducing the dimension of the optimization problem to be solved and the computational complexity of its implementation. The optimality criterion of routing solutions contributes to the formation of primary and backup disjoint paths with the maximum bandwidth. Additionally, the path with the highest bandwidth will correspond to the primary path, while the rest of the paths will be used as a backup in decreasing order of their bandwidth.
\end{abstract}

Keywords: Fast ReRouting; path protection; disjoint paths; bandwidth; optimization.

\section{INTRODUCTION}

As is known, while ensuring the fault tolerance of the infocommunication network (ICN) at the network level, the key role is given to Fast ReRoute (FRR) protocol solutions $[1,2]$. Thus, for the transmission of packet flows the primary and backup routes are calculated with the protection of the Quality of Service (QoS) along them by such QoS indicators as bandwidth, average delay and the probability of packet loss $[3,4]$. In addition, the implementation of FRR also raises the problem of calculating the set of disjoint paths $[5,6]$. This formulation of the problem meets the requirements of increasing the fault tolerance of routing solutions, especially when the protection of paths and their bandwidth is required. In this regard, the task of development of the FRR model with the implementation of a path protection scheme and bandwidth is relevant. In addition, the proposed model should provide scalability of the obtained solutions and low computational complexity of its further protocol implementation.

\section{FAST REROUTING FLOW-BASED MODEL WITH IMPLEMENTATION OF PATH PROTECTION}

The paper proposes the FRR flow-based model with the implementation of the path and its bandwidth protection presented by additional conditions. The introduction of these conditions allows reducing the solution of the technological FRR task to the solution of the optimization problem of mixed integer linear programming with a modified optimality criterion that is introduced for inclusion in the calculated routes of links with high bandwidth. The result of solving the formulated optimization problem is the calculation of the set of disjoint routes. Of this set, the route with high bandwidth will correspond to the primary path, while other routes will be used as backup routes in decreasing order of their bandwidth. Thus, each of the calculated routes will have the required bandwidth.

The advantages of the proposed model include the fact that the implementation of the $1: n$ path protection scheme does not lead to a proportional increase in the dimension of the optimization problem. The optimality criterion used is aimed at the fact that the set of calculated paths contains routes that not only meet the bandwidth requirements but also include the most productive communication links. The linearity of the proposed flow-based FRR model and the reduction of the number of routing variables to be calculated helped to decrease the complexity of its computational implementation during the organization of fast routing on the network.

\section{CONCLUSIONS}

In the course of the study, a comparison of the routing solutions that were obtained using the FRR model with the optimality criteria for different network structures and redundancy schemes $-1: 2$ and 1:3 has been conducted. The prospect of further research in this area is primarily concerned with the support of multipath routing strategies, as well as the implementation of schemes to protect QoS indicators (bandwidth, average delay, jitter, packet loss probabilities), and Quality of Experience (QoE) indicators, such as rating and multimedia quality.

\section{REFERENCES}

[1] J. Rak, Resilient Routing in Communication Networks. 1st edition, Springer, 2015.

[2] D. Tipper, "Resilient network design: challenges and future directions," in Telecommunication Systems, vol. 56, iss. 1, 2014, pp. 5-16.

[3] O. Lemeshko, M. Yevdokymenko and O. Yeremenko, "Model of data traffic QoS fast rerouting in infocommunication networks," Innovative Technologies and Scientific Solutions for Industries, Vol. 3, Iss. 9, pp. 127-134.

[4] L. Guo, "Efficient approximation algorithms for computing $\mathrm{k}$ disjoint constrained shortest paths," Journal of Combinatorial Optimization, Vol. 32, Iss. 1, 2016, pp. 144-158.

[5] O. Lemeshko, O. Yeremenko, M. Yevdokymenko, B. Sleiman, A.M. Hailan and A. Mersni, "Computation Method of Disjoint Paths under Maximum Bandwidth Criterion", in Proc. 3rd IEEE International Conference Advanced Information and Communication Technologies (AICT), 2019, pp. 161-164.

[6] P. Cruz, T. Gomes and D. Medhi, "A Heuristic for Widest Edge-disjoint Path Pair Lexicographic Optimization," in Proc. 2014 6th International Workshop on Reliable Networks Design and Modeling, 2014, pp. 